\title{
Artificial fish swarm optimization algorithm for power system state estimation
}

\author{
Surender Reddy Salkuti \\ Department of Railroad and Electrical Engineering, Woosong University, Republic of Korea
}

\begin{tabular}{l} 
Article Info \\
\hline Article history: \\
Received Sep 26, 2019 \\
Revised Nov 27, 2019 \\
Accepted Dec 11, 2019 \\
\hline
\end{tabular}

\section{Keywords:}

Evolutionary algorithm

Load flow

Pseudo measurements

State estimation

\begin{abstract}
In this paper, the power system state estimation (SE) problem is formulated as a general non-linear programming problem with equality constraints and boundary limits on the state variables. The proposed SE problem is solved using an evolutionary based Artificial Fish Swarm Optimization Algorithm (AFSOA). The AFSOA is a global search algorithm based on the characteristics of fish swarm and its autonomous model. The detailed algorithm with its flow chart is presented in this paper. To show the effectiveness of the proposed SE approach, six bus test system is considered. The obtained results are compared with other algorithms reported in the literature.
\end{abstract}

\section{Corresponding Author:}

Surender Reddy Salkuti, Department of Railroad and Electrical Engineering, Woosong University, Daejeon, Republic of Korea.

Email: surender.wsu@gmail.com

\section{NOMENCLATURE}

\begin{tabular}{|c|c|c|c|}
\hline $\begin{array}{l}\mathrm{N} \\
\mathrm{Z}\end{array}$ & $\begin{array}{l}\text { Number of buses in the system. } \\
\text { Measurement vector. }\end{array}$ & $\begin{array}{l}\mathrm{Q}_{\mathrm{Gi}}^{\min }, \mathrm{Q}_{\mathrm{Gi}}^{\max } \\
\text { generation lim }\end{array}$ & $\begin{array}{l}\text { Minimum and maximum reactive power } \\
\text { it of } i^{\text {th }} \text { generator. }\end{array}$ \\
\hline$\epsilon$ & Measurement noise vector, & artificial fish. & \\
\hline $\mathrm{P}_{\mathrm{li}}^{\max }$ & Maximum active power flow in line-i. & $\mathrm{X}$ & State vector. \\
\hline $\mathrm{Qi}_{\mathrm{i}}$ & Reactive power injection at $\mathrm{i}^{\text {th }}$ bus. & $X_{i}$ & Position within the scope of the vision of \\
\hline$Y_{\mathrm{ij}}$ & $\mathrm{G}_{\mathrm{ij}}+\mathrm{j} \mathrm{B}_{\mathrm{ij}}=\mathrm{i}-\mathrm{j}^{\text {th }}$ element of $\mathrm{Y}$-bus matrix. & $\mathrm{n}_{\mathrm{f}}$ & Number of artificial fish. \\
\hline $\mathrm{N}_{\mathrm{l}}$ & Number of load buses. & $X_{c}$ & Center of the swarm. \\
\hline $\mathrm{N}_{\mathrm{g}}$ & Number of generator buses. & $\mathrm{d}_{\text {visual }}$ & Perceiving range of an artificial fish. \\
\hline
\end{tabular}

\section{INTRODUCTION}

Load flow calculations indeed are an inevitable tool for off-line studies and planning exercises, but incomplete and erroneous measurement is a real time proposition. The solution for such a situation is provided by static state estimator, which ignores the slow changes in the system and utilizes redundant set of measurements for cross checking and approximating the most reliable estimates of the system state. State estimation (SE) processes a set of measurements to obtain the best estimate of current state of power system. The state estimator plays an essential role of a purifier, creating a complete and reliable database for security monitoring, security analysis and the various controls of a power system. Therefore, the state 
estimator employs statistical methods to act as a tunable filter between the field data measurements and the security and control functions. The set of measurements includes telemetered measurements and pseudomeasurements. The telemetered measurements are the online telemetered data of bus voltages, line flows, injections, etc. The pseudo-measurements are the manufactured data such as guessed MW generation or substation load demand based on historical data. Telemetered measurements are subject to noise or error in metering, communication system, etc. The errors of some of the pseudo-measurements, especially the guessed ones, may be large. However, there is a special type of pseudo-measurements, known as the zero injections, for which the information contains no error [1].

The conventional form of solving the SE, i.e., the weighted least square (WLS) method doesn't enforce the equality and limit constraints explicitly. However, the constraints contain reliable information about physical restrictions and equipment limits and can be used to increase the quality of SE result. The zero injections can be represented by a set of equalities. In the literature, various methods have been proposed to process constraints, and for solving the equality constrained SE problem [2].

A hybrid state estimator that consists of a cellular computational network and genetic algorithm (GA) is proposed in Reference [3]. A practical approach to incorporate the FACTS controllers into a Weighted Least Squares (WLS) SE algorithm is proposed in [4]. In Reference [5], a new algorithm based on node voltage method, Kron reduction matrix, modeling of power system in frequency domain using phase values and GA optimization is proposed for the harmonic SE in power systems. A new approach for the SE of a non-linear AC power system in a non-iterative manner based on the Kipnis-Shamir relinearization technique is proposed in [6]. The application of an artificial intelligence based particle swarm optimization (PSO) algorithm is used in [7] to solve the SE problem within a power system. A robust least winsorized square estimator is proposed in [8] for solving the static SE problem of power system. A re-weighted moving horizon estimation technique is proposed in [9] for the robust power system SE, which adaptively estimates the system states along with an updated noise variance.

A novel robust least winsorized square estimator for dynamic SE of a power system is proposed in [10]. A SE approach which results in the formation of constant Jacobian matrix has been proposed in [11], and it has been solved using Biogeography-based optimization (BBO) algorithm for various percentages of bad measurements. A decentralized state estimator for large-scale interconnected systems is proposed in [12]. Reference [13] demonstrates the performance of the estimator for experimental flight data and compare it to a state-of-the-art estimator based on inertial measurements. An approach for static SE of power system parameters is proposed in [14]. Reference [15] solves an optimal filtering problem for distributed dynamic systems. Reference [16] uses the maximum likelihood ensemble filter for the estimation of the states of a power system. A decentralized approach for the SE of power system by casting it in the framework of multiagent optimization is proposed in [17]. Reference [18] proposes a power system SE algorithm in the presence of irregular sensor sampling and random communication delays. Reference [19] proposes a novel feasible point pursuit based SE solver, which iteratively seeks feasible solutions for a non-convex quadratically constrained quadratic programming reformulation of the WLS SE problem.

In this paper, a new method for enforcing the equality and limit constraints in state estimation (SE) problem using Artificial fish swarm optimization algorithm (AFSOA) is proposed. The AFSOA is a novel bionic optimization technique, which searches for an optimal solution in the target solution space by simulating fish preying, swarming, random and following behaviors. The main advantages of using the AFSOA is that it uses the target function as an algorithm evaluation function directly and it can be used to get the appropriate solution quickly.

\section{STATE ESTIMATION (SE): PROBLEM FORMULATION}

The state vector of an electric network consists of the complex voltages at the buses. The unmeasured tap positions of transformers may also be included into the state vector. A measurement vector consists of power flows, power injections, voltage and current magnitudes and tap positions of transformers. For a $N$ bus system, the state vector $(\mathrm{x})$ can be represented by $\mathrm{x}=[\delta, \mathrm{V}]^{\mathrm{T}}$, of dimension $\mathrm{n}=2 \mathrm{~N}-1$, consists of the N-1 bus voltage angles $\left(\delta_{\mathrm{i}}\right)$ with respect to a reference bus, and the $N$ bus voltage magnitudes $\mathrm{V}_{\mathrm{i}}$ for $\mathrm{i}=1,2,3, \ldots, \mathrm{N}$. The general static state estimator measurement model is represented by [20],

$$
z=h(x)+\epsilon
$$

where $\mathrm{z}$ represents all measurements, including the power injection, power flow and bus voltage magnitude measurements. $x$ is the state vector composed of phase angles and magnitudes of the voltages at network buses, and $h($.$) stands for the nonlinear measurement functions in terms of state variables [21].$ 
The model of network constitutes its topology and parameters. The topological errors can be caused by missing information in respect of disconnected or reconnected line, while parameter errors are due to its wrong initial estimation. Moreover, the system representation considered in such studies is single phase, while unbalance conditions cause significant error. Non-availability of measurements may create condition of unobservability and therefore it is important to maintain sufficient redundancy. In this paper, the error-free data are modeled as equality constraints [22], and

$$
g(x)=0
$$

Limits on some network variables are modeled as inequality constraints which can be expressed in a compact form by p-dimensional functional inequalities.

$$
f(x) \leq 0
$$

General nonlinear programming algorithms for the solution of a constrained minimization problem are not efficient enough for the on-line application. Hence, an evolutionary based technique is used for solving this nonlinear programming problem [23]. The estimate of state vector (x) can be obtained by minimizing the weighted least square (WLS) function, and it is expressed as,

$$
J(x)=[z-h(x)]^{T} W[z-h(x)]
$$

where $\mathrm{W}$ is a diagonal matrix of size $(m \times m)$, whose elements are the measurement weighting factors. The measurement weighting factor is taken as the reciprocal of the error variance. The estimate is solved by an iterative scheme, which computes the corrections $(\Delta \mathrm{x})$ at each iteration by solving the following function [24],

$$
G(x) \Delta x=H(x)^{T} W \Delta z
$$

where $\Delta \mathrm{z}=z-h(x)$, and $\mathrm{H}(\mathrm{x})=\frac{\partial h(x)}{\partial x}=\mathrm{Jacobian}$ matrix.

$$
G(x)=H(x)^{T} W H(x)
$$

where $\mathrm{x}=\mathrm{x}^{\mathrm{k}}$ at the $\mathrm{k}^{\text {th }}$ iteration.

In the AC network, the measured quantities are active power (MW), reactive power (MVAR), apparent power (MVA), current (A), transformer tap position, and voltage magnitudes. The state variables are the voltage magnitude at each bus and the phase angles at all buses except the reference bus. The equation for power flowing over a transmission line is clearly not a linear function of the voltage magnitude and phase angle at each end of line. Therefore, the $\mathrm{h}(\mathrm{x})$ functions will be nonlinear functions; except for a voltage magnitude measurement where $\mathrm{h}(\mathrm{x})$ is simply unity times the particular $\mathrm{x}_{\mathrm{i}}$ that corresponds to the voltage magnitude being measured [25-26].

\subsection{Formulation of Objective Function}

The objective is to minimize the weighted squared mismatch between the measured and the calculated quantities. Considering system to be observable and with $(m>n)$ condition, then the mathematical problem is expressed as [27],

$$
\text { minimize } \quad \frac{1}{2}[z-h(x)]^{T} R^{-1}[z-h(x)]
$$

where the diagonal matrix $\left(R^{-1}\right)$ represents the weights of the individual measurements in the objective function. As shown in (7) is solved subject to the following equality and inequality constraints.

\subsection{Equality constraints}

The power flow equations, corresponding to both active and reactive power balances are the equality constraints for all the buses characterized as zero injections, which can be expressed as [28],

$$
\begin{aligned}
& P_{i}=\sum_{m=1}^{N} V_{i} V_{m}\left(G_{i m} \cos \delta_{i m}+B_{i m} \sin \delta_{i m}\right)=0 \\
& Q_{i}=\sum_{m=1}^{N} V_{i} V_{m}\left(G_{i m} \sin \delta_{i m}-B_{i m} \cos \delta_{i m}\right)=0
\end{aligned}
$$

for $\mathrm{i} \epsilon$ (set of zero injection buses). 


\subsection{Inequality Constraints}

Voltage Constraints: This include upper $\left(V_{i}^{\max }\right)$ and lower $\left(V_{i}^{\min }\right)$ limits on the bus voltage magnitude [29], and they are expressed as,

$$
V_{i}^{\min } \leq V_{i} \leq V_{i}^{\max } \quad i=1,2,3, \ldots \ldots, N
$$

Phase Angle Constraints: The phase angle at each bus should be between lower $\left(\delta_{\mathrm{i}}^{\mathrm{min}}\right)$ and upper $\left(\delta_{\mathrm{i}}^{\mathrm{max}}\right)$ limits, and they are expressed as,

$$
\delta_{i}^{\min } \leq \delta_{i} \leq \delta_{i}^{\max } \quad i=1,2,3, \ldots \ldots, N
$$

The voltage and phase angle constraints may vary depending upon the problem under consideration. Imposing the phase angle constraints at load buses is another way of limiting the power flow in the transmission lines and for generator buses this limiting is done for stability reasons.

Line Flow Constraints: They represent the maximum power flow in a transmission line and is usually based on thermal and dynamic stability considerations. The line flow constraint can be expressed as,

$$
P_{l i}^{\max } \geq P_{l i} \quad i=1,2,3, \ldots \ldots, N_{l}
$$

Generator Reactive Power Constraint: The reactive power limits of generator are expressed as,

$$
Q_{G i}^{\min } \leq Q_{G i} \leq Q_{G i}^{\max } \quad i=1,2,3, \ldots \ldots, N_{g}
$$

\section{RESEARCH METHOD}

The SE problem formulated in this paper is solved by using the Artificial fish swarm optimization algorithm (AFSOA). The AFSOA is a meta-heuristic/evolutionary based algorithm, which is a comparatively topical accumulation to the pasture of natural computing, that has rudiments enthused by the societal behaviors of natural swarms, and associates with evolutionary computation. It has an extensive application in multifaceted optimization domains, and currently a foremost research focus, contribution an unconventional to the more established meta-heuristic techniques that may applied in many of the identical domains [30]. AFSOA has several characteristics that are similar to genetic algorithm (GA) such as sovereignty from incline in sequence of purpose occupation, the capability to resolve multifaceted nonlinear high dimensional exertion [30]. Furthermore, they canister accomplish closer convergence swiftness and entail the minority parameters to bend. Whereas, the AFSOA does not seize the crossover and mutation processes utilize in GA, so it could achieve more simply.

In nature, the fish can find out the more nutritious area by individual search or following other fish, the area with much more fish is commonly most nutritious. The fundamental idea of the AFSOA is to reproduce the fish behaviors such as praying, swarming, and following with local search of fish individual for attaining the global optimum. Fish habitually reside within the place having a lot of food. Therefore, the behaviors of fish is imitated based on this attribute to come across the global optimum, which is the indispensable inspiration of the AFSOA. In this paper, the artificial fish denotes the decision variables used in the optimization problem. The power output from each generating unit forms the artificial fishes. Various steps that are involved for the implementation of AFSOA is described next.

\subsection{Initialization}

The position of each artificial fish denotes a possible potential solution. They are the decision variables used in the optimization problem. The current position $\left(X_{i}\right)$ can be represented as [31],

$$
X_{i}=\left(X_{i 1}, X_{i 2}, X_{i 3}, \ldots, X_{i n}\right)
$$

where $i$ is number control variables, $X_{i}$ denotes an initial solution, $n$ is number fishes in the swarm (i.e., swarm length).

\subsection{Fitness Function Evaluation}

The fitness function (FF) is formulated as a function of current position $\left(X_{i}\right)$ and it is expressed as,

$$
F F_{i}=f\left(X_{i}\right)
$$

The position is defined by different behaviors of fish and they are described as follows: 


\subsection{Preying Behavior}

This fundamental biological behavior tends to the food. Let the condition of artificial fish is $X_{i}$, choosing a state $X_{j}$ inside its sensing range arbitrarily. Suppose $X_{j}$ is superior to $X_{i}$, then move to $X_{j}$, otherwise to the selected arbitrarily condition $X_{i}$. Determine whether to meet the forward conditions, repeated several times, if still not satisfied forward conditions, then move one step randomly. The food concentration in this position of fish is stated as the objective function value. The distance between the artificial fish is [32]-[33] expressed as,

$$
\begin{aligned}
& d_{i j}=\left\|X_{i}-X_{j}\right\| \\
& X_{j}=X_{i}+\left(d_{\text {visual }} \text {.rand }()\right)
\end{aligned}
$$

where $d_{\text {visual }}$ is the distance through which the artificial fish can see (i.e., perceiving range/visual distance) [34], and $\operatorname{rand}()$ produces random numbers between 0 and 1.

$$
X_{i}^{(t+1)}=X_{i}^{t}+\left(\frac{X_{j}-X_{i}^{t}}{\left\|X_{i}-X_{i}^{t}\right\|} \cdot \text { step } \cdot \operatorname{rand}()\right)
$$

where step is the maximum distance that a fish can move in one movement.

\subsection{Swarming Behavior}

Let the current position of artificial fish is $X_{i}$ and $\left(d_{i j}<d_{v i s u a l}\right)$. Then, $\left(n_{f}<\delta\right)$ indicates that the partners have more food and less crowded. Artificial fish has a tendency to move towards the center of the swarm, to ensure the presence of swarm around it and to avoid any potential danger. Now, the updated position is given by [35],

$$
X_{i}^{(t+1)}=X_{i}^{t}+\left(\frac{X_{c}-X_{i}^{t}}{\left\|X_{c}-X_{i}^{t}\right\|} \cdot \text { step.rand }\right)
$$

\subsection{Following Behavior}

Let the state of artificial fish is $X_{i}$ exploring its optimal state $X^{\text {opt }}$ from the visual neighbors. Suppose, the number of partners of $X^{\text {opt }}$ is $\left(n_{f}<\delta\right)$ indicates that near distance have more food, not too crowed and can move further. Otherwise, perform the prey behavior by using the (13) [35]. The flow chart of Artificial fish swarm optimization algorithm (AFSOA) is depicted in Figure 1 [36].

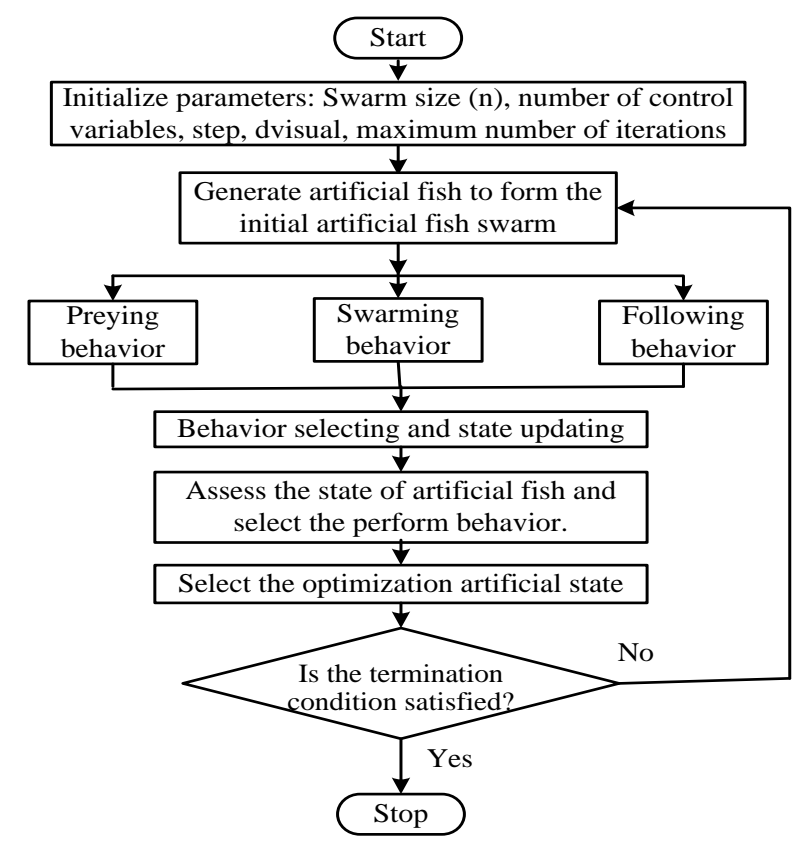

Figure 1. Flow Chart of Artificial fish swarm optimization Algorithm (AFSOA) 


\section{RESULTS AND DISCUSSION}

In this paper, 6 bus [37] system is used for solving the proposed state estimation (SE) problem. The voltage magnitudes at each bus and the phase angles at all the buses except the reference bus are considered as the state variables for the SE problem. The true values were obtained by the result of the load flow calculation, and the measurement values were made by adding errors to those true values. As equality constraints, zero power injection at the nodes with no load and no generation are used. As mentioned earlier, here, the AFSO algorithm is used to solve the proposed SE problem. The parameters considered in this paper are: Population size is 60, step is 1 and the maximum number of iterations are 200. The data required for solving the SE problem on 6 bus system is taken from Reference [37]. The measurement set data of 6 bus system is depicted in Figure 2 and in Table 1. The bus numbers 3 and 4 are characterized as zero injection buses. In the 6 bus system, total 15 measurements are considered, in that 4 are considered as the bus injection type measurements and 11 are considered as the line flow type measurements. With this data, the SE is performed by using the AFSOA [38]. The estimated state variables by solving the SE with equality constraints are presented in Table 2.

The errors of the estimate values (i.e., $\Delta \mathrm{P}$ and $\Delta \mathrm{Q}$ ) obtained by solving the SE using AFSOA are presented in Table 3. The errors of estimated values obtained using the AFSOA are also compared with the conventional WLS technique and the evolutionary based PSO algorithm.

From the above simulation results, it can be observed that by solving the SE problem (with the equality constraints) using Artificial fish swarm optimization algorithm (AFSOA) has better results compared to conventional weighted least square (WLS) technique and the evolutionary based particle swarm optimization (PSO) algorithm.

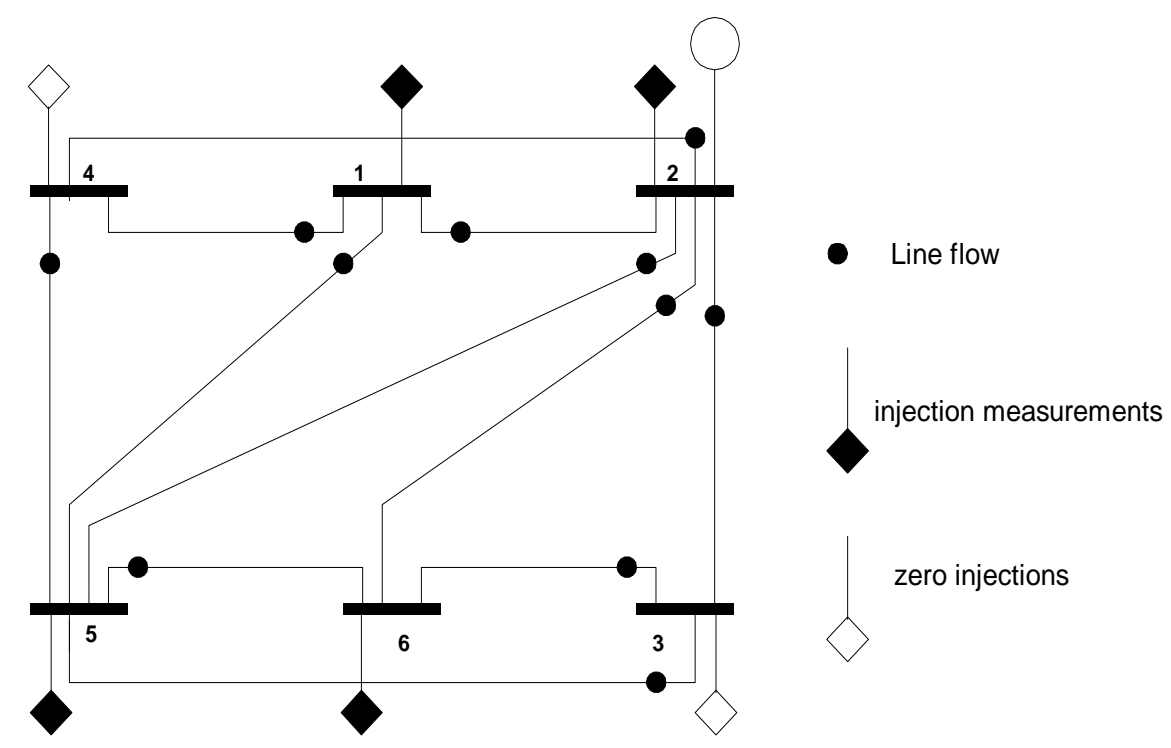

Figure 2. Measurement data set for 6 bus system

Table 1. Measurement (Bus Injection and Line Flow Type Measurements) Data Set for 6 Bus System

\begin{tabular}{ccccc}
\hline Measurements & Type & Buses & $\mathrm{P}(\mathrm{MW})$ & $\mathrm{Q}(\mathrm{MW})$ \\
\hline $\mathrm{Z}_{1}$ & Injection & 1 & 97.40 & -6.61 \\
$\mathrm{Z}_{2}$ & Injection & 2 & 50.05 & 50.75 \\
$\mathrm{Z}_{3}$ & Injection & 5 & -70.07 & -70.07 \\
$\mathrm{Z}_{4}$ & Injection & 6 & -70.07 & -70.07 \\
$\mathrm{Z}_{5}$ & Line flow & $1-2$ & 28.80 & -15.50 \\
$\mathrm{Z}_{6}$ & Line flow & $1-4$ & 28.30 & -8.80 \\
$\mathrm{Z}_{7}$ & Line flow & $1-5$ & 40.10 & 17.60 \\
$\mathrm{Z}_{8}$ & Line flow & $2-3$ & 23.10 & 19.40 \\
$\mathrm{Z}_{9}$ & Line flow & $2-4$ & -9.0 & -7.00 \\
$\mathrm{Z}_{10}$ & Line flow & $2-5$ & 20.60 & 21.10 \\
$\mathrm{Z}_{11}$ & Line flow & $2-6$ & 43.20 & 4.40 \\
$\mathrm{Z}_{12}$ & Line flow & $3-5$ & 1.10 & 5.20 \\
$\mathrm{Z}_{13}$ & Line flow & $3-6$ & 21.50 & 18.10 \\
$\mathrm{Z}_{14}$ & Line flow & $4-5$ & 18.90 & 9.00 \\
$\mathrm{z}_{15}$ & Line flow & $5-6$ & 7.3 & -4.4 \\
\hline
\end{tabular}


Table 2. Estimated State Variables After Solving the SE with Equality Constraints for 6 Bus System

\begin{tabular}{ccccccc}
\hline Bus number & 1 & 2 & 3 & 4 & 5 & 6 \\
\hline Bus voltage $(\mathrm{V})$ & 1.0503 & 1.0494 & 0.9892 & 1.0503 & 0.9656 & 0.9683 \\
Phase angle $(\delta)$ & 0 & -4.7065 & -7.6059 & -3.8441 & -6.9388 & -8.8593 \\
\hline
\end{tabular}

Table 3. Errors of Estimated Values Obtained by Solving the SE using WLS Technique, PSO and AFSOA

\begin{tabular}{ccccccc}
\hline & \multicolumn{2}{c}{ SE Using WLS Technique } & \multicolumn{2}{c}{ SE Using PSO } & \multicolumn{2}{c}{ SE Using AFSOA } \\
Measurements & $\Delta \mathrm{P}$ & $\Delta \mathrm{Q}$ & $\Delta \mathrm{P}$ & $\Delta \mathrm{Q}$ & $\Delta \mathrm{P}$ & $\Delta \mathrm{Q}$ \\
\hline $\mathrm{Z}_{1}$ & -0.026 & 0.0054 & -0.025 & 0.0054 & -0.021 & 0.0051 \\
$\mathrm{Z}_{2}$ & 0.0069 & 0.005 & 0.0068 & 0.0005 & 0.0068 & 0.0005 \\
$\mathrm{Z}_{3}$ & 0.0039 & -0.0003 & 0.0039 & -0.0003 & 0.0037 & -0.0003 \\
$\mathrm{Z}_{4}$ & 0.0078 & -0.0094 & 0.0078 & -0.0095 & 0.0077 & -0.0093 \\
$\mathrm{Z}_{5}$ & -0.0085 & 0.0009 & -0.0083 & 0.0009 & -0.0083 & 0.0008 \\
$\mathrm{Z}_{6}$ & -0.0069 & -0.0014 & -0.0069 & -0.0014 & -0.0068 & -0.0013 \\
$\mathrm{Z}_{7}$ & -0.0064 & -0.0022 & -0.0062 & -0.0023 & -0.006 & -0.0022 \\
$\mathrm{Z}_{8}$ & -0.0022 & -0.0015 & -0.0022 & -0.0015 & -0.0021 & -0.0014 \\
$\mathrm{Z}_{9}$ & 0.0048 & -0.0133 & 0.0047 & -0.0132 & 0.0046 & -0.0131 \\
$\mathrm{Z}_{10}$ & -0.0001 & -0.0017 & -0.0001 & -0.0017 & -0.0001 & -0.0016 \\
$\mathrm{Z}_{11}$ & -0.0033 & -0.0233 & -0.0033 & -0.0232 & -0.0033 & -0.0233 \\
$\mathrm{Z}_{12}$ & 0.0013 & -0.0039 & 0.0013 & -0.0038 & 0.0012 & -0.0038 \\
$\mathrm{Z}_{13}$ & -0.0028 & 0.0021 & -0.0028 & 0.0021 & -0.0027 & 0.002 \\
$\mathrm{Z}_{14}$ & -0.0011 & -0.0037 & -0.0011 & -0.0037 & -0.0011 & -0.0036 \\
$\mathrm{Z}_{15}$ & -0.0019 & -0.0007 & -0.0019 & -0.0007 & -0.0019 & -0.0007 \\
\hline
\end{tabular}

\section{CONCLUSION}

In this paper, the power system state estimation (SE) problem is formulated as a general non-linear programming problem with equality constraints and boundary limits on the state variables. By enforcing the limits on bus voltages, phase angles, line flows and reactive power generations, and equality constraints increases the quality of SE results and produces the solution, which reflects correctly the physical behavior of a power system. The presented SE problem is solved using the Artificial Fish Swarm Optimization Algorithm (AFSOA). AFSOA is a novel bionic optimization technique, which searches for an optimal solution in the target solution space by simulating fish preying, swarming, random and following behaviors. The main advantages of using the AFSOA is that it directly uses the target function as an algorithm evaluation function and it can be used to get the appropriate solution quickly.

\section{ACKNOWLEDGEMENTS}

This research work has been carried out based on the support of "Woosong University's Academic Research Funding - (2019-2020)".

\section{REFERENCES}

[1] F. Schmidt, M.C. de Almeida, "A theoretical framework for qualitative problems in power system state estimation, Electric Power Systems Research, vol. 154, pp. 528-537, 2018.

[2] D.H. Tungadio, J.A. Jordaan, M.W. Siti, "Power system state estimation solution using modified models of PSO algorithm: Comparative study", Measurement, vol. 92, pp. 508-523, 2016.

[3] Md.A. Rahman, G.K. Venayagamoorthy, "A hybrid method for power system state estimation using Cellular Computational Network", Engineering Applications of Artificial Intelligence, vol. 64, pp. 140-151, 2017.

[4] A.Z. Cárdenas, C.R.F. Esquivel, "State estimation of power systems containing facts controllers", Electric Power Systems Research, vol. 81, no. 4, pp. 995-1002, 2011.

[5] V. Bečirović, I. Pavić, B.F. Grčić, "Sensitivity analysis of method for harmonic state estimation in the power system", Electric Power Systems Research, vol. 154, pp. 515-527, 2018.

[6] X.T. Jiang, J.H. Chow, B. Fardanesh, D. Maragal, G. Stefopoulos, M. Razanousky, "Power system state estimation using a direct non-iterative method", International Journal of Electrical Power \& Energy Systems, vol. 73, pp. 361$368,2015$.

[7] D.H. Tungadio, B.P. Numbi, M.W. Siti, A.A. Jimoh, "Particle swarm optimization for power system state estimation", Neurocomputing, vol. 148, pp. 175-180, 2015.

[8] B. Vedik, A.K. Chandel, "Power system static state estimation using a least winsorized square robust estimator", Neurocomputing, vol. 207, pp. 457-468, 2016.

[9] T. Chen, "Robust state estimation for power systems via moving horizon strategy", Sustainable Energy, Grids and Networks, vol. 10, pp. 46-54, 2017.

[10] V. Basetti, A.K. Chandel, R. Chandel, "Power system dynamic state estimation using prediction based evolutionary technique", Energy, vol. 107, pp. 29-47, 2016. 
[11] M. Kalpanadevi, R. Neela, "BBO Algorithm for Line Flow Based WLS State Estimation", Materialstoday: Proceedings, vol. 5, no. 1, pp. 318-328, 2018.

[12] H. Liu, H. Yu, "Decentralized state estimation for a large-scale spatially interconnected system", ISA Transactions, vol. 74, pp. 67-76, 2018.

[13] M. Polzin, T.A. Wood, H. Hesse, R.S. Smith, "State Estimation for Kite Power Systems with Delayed Sensor Measurements", IFAC-PapersOnLine, vol. 50, no. 1, pp. 11959-11964, 2017.

[14] A. Aboonabi, M. Saif, "Power System State Estimation Using Contraction Mapping and Singular Value Decomposition", IFAC Proceedings Volumes, vol. 44, no. 1, pp. 1692-1697, 2011.

[15] Md.M. Rana, L. Li, S.W. Su, "Distributed State Estimation for Interconnected Synchronous Generators", IFACPapersOnLine, vol. 50, no. 1, pp. 5418-5423, 2017.

[16] B. Uzunoğlu, M.A. Ülker, "Maximum Likelihood Ensemble Filter State Estimation for Power Systems", IEEE Trans. on Instrumentation and Measurement, vol. 67, no. 9, pp. 2097-2106, 2018.

[17] A. Konar, A.S. Zamzam, N.D. Sidiropoulos, "Decentralized power system state estimation via non-convex multiagent optimization", IEEE Global Conference on Signal and Information Processing, Montreal, QC, 2017, pp. 1060-1064.

[18] A.M. Stanković, V. Švenda, A.T. Sarić, M.K. Transtrum, "Hybrid power system state estimation with irregular sampling", IEEE Power \& Energy Society General Meeting, Chicago, IL, 2017, pp. 1-5.

[19] G. Wang, A.S. Zamzam, G.B. Giannakis, N.D. Sidiropoulos, "Power system state estimation via feasible point pursuit", IEEE Global Conference on Signal and Information Processing, Washington, DC, 2016, pp. 773-777.

[20] T. Khadour, M.A. Saba, L. Saleh, "Improving bearings-only target state estimation tracking problem by using adaptive and nonlinear kalman algorithms", Indonesian Journal of Electrical Engineering and Computer Science, vol. 15, no. 1, pp. 190-198, Jul. 2019.

[21] M.N. Kamarudin, T.J.T. Hashim, A.H. Musa, "Optimal sizing and location of distributed generation for loss minimization using firefly algorithm", Indonesian Journal of Electrical Engineering and Computer Science, vol. 14, no. 1, pp. 421-427, Apr. 2019.

[22] J.P. Sridhar, R. Prakash, "Multi-objective whale optimization based minimization of loss, maximization of voltage stability considering cost of DG for optimal sizing and placement of DG", International Journal of Electrical and Computer Engineering (IJECE), vol. 9, no. 2, pp. 835-839, Apr. 2019.

[23] O. Olatunde, H.A. Rahman, "Allocation of distributed generation and capacitor banks in distribution system", Indonesian Journal of Electrical Engineering and Computer Science, vol. 13, no. 2, pp. 437-446, Feb. 2019.

[24] S.M. Hossain, A.H. Chowdhury, Multi-objective optimal placement of distributed generations for dynamic loads, International Journal of Electrical and Computer Engineering (IJECE), vol. 9, no. 4, pp. 2303-2313, Aug. 2019.

[25] S.C. Kim, S.R. Salkuti, "Optimal power flow based congestion management using enhanced genetic algorithms", International Journal of Electrical and Computer Engineering (IJECE), vol. 9, no. 2, pp. 875-883, Apr. 2019.

[26] P.L. Reddy, G. Yesuratnam, "A modified bacterial foraging algorithm based optimal reactive power dispatch", Indonesian Journal of Electrical Engineering and Computer Science, vol. 13, no. 1, pp. 361-367, Jan. 2019.

[27] G. Wang, A.S. Zamzam, G.B. Giannakis, N.D. Sidiropoulos, "Power System State Estimation via Feasible Point Pursuit: Algorithms and Cramér-Rao Bound", IEEE Transactions on Signal Processing, vol. 66, no. 6, pp. 16491658, 2018.

[28] M. Farsadi, F.M. Shahir, E. Babaei, "Power system states estimations using Kalman filter", 10th International Conference on Electrical and Electronics Engineering, Bursa, pp. 808-812, 2017.

[29] P. Puviya, N. Priyadarshini, "State estimation in power system using weighted least squares method", International Conference on Innovations in Information, Embedded and Communication Systems, Coimbatore, 2017, pp. 1-6.

[30] X.Y. Luan, Z.P. Li, T.Z. Liu, "A novel attribute reduction algorithm based on rough set and improved artificial fish swarm algorithm", Neurocomputing, vol. 174, no. A, pp. 522-529, 2016.

[31] N. Fang, J. Zhou, R. Zhang, Y. Liu, Y. Zhang, "A hybrid of real coded genetic algorithm and artificial fish swarm algorithm for short-term optimal hydrothermal scheduling", Electrical Power and Energy Systems, vol. 62, pp. 617$629,2014$.

[32] K.P. Kumara, B. Saravanana, K.S. Swarup, "Optimization of Renewable Energy Sources in a Microgrid Using Artificial Fish Swarm Algorithm", Energy Procedia, vol. 90, pp. 107-113, 2016.

[33] Q. He, X. Hub, H. Ren, H. Zhang, "A novel artificial fish swarm algorithm for solving large-scale reliabilityredundancy application problem", ISA Transactions, vol. 59, pp. 105-113, 2015.

[34] Y. Wang, "Artificial Fish Swarm Algorithm based Optimization of Load Dispatch Problem for GTCC Units", International Journal of Engineering Research \& Technology, vol. 5, no. 1, pp. 340-344, 2016.

[35] Y. Tian, C. Lu, Z. Wang, L. Tao, "Artificial Fish Swarm Algorithm-Based Particle Filter for Li-Ion Battery Life Prediction", Hindawi Publishing Corporation, Mathematical Problems in Engineering, vol. 204, pp. 1-10, 2014.

[36] K.J. Seog, S.S. Reddy, C.M. Jung, "Solving Non-convex Economic Dispatch with Prohibited Zones using Artificial Fish Swarm Optimization", International Journal of Engineering \& Technology, vol. 7, no. 2.18, pp. 1-6, Apr. 2018.

[37] A.J. Wood, B.F. Wollenberg, Power Generation, Operation and Control, 3rd Edition, John Wiley and Sons, New Jersey, USA, Dec. 2013.

[38] S.S. Reddy, "Solving the Power System State Estimation Problem Embedded with UPFC Using Glowworm Swarm Optimization Algorithm", International Journal of Applied Engineering Research, vol. 12, no. 10, pp. 2361-2369, Jun. 2017. 